\title{
Stability Analysis of the Endemic Equilibrium State on the Spread of Malaria Using Bellman and Cooke's Theorem
}

\author{
Musa, BAWA \\ Department of Mathematics/Computer Science, Ibrahim Badamasi Babangida University, Lapai, Nigeria.
}

\begin{abstract}
Malaria is a life threatening blood disease which is caused by parasites transmitted to humans through the bite of the Anopheles mosquito. An infected mosquito bites a human and transmits the parasites which multiply in the host's liver before infecting and destroying red blood cells. In this research work, the model equations were obtained using several known clinical and biological information with the human population subdivided into susceptible, infected, recovered and therapy classes while the vector population is divided into the susceptible and infected classes. The equilibrium states were obtained and the endemic state analysed for stability. The result shows that the non-zero equilibrium state will be stable if $F(0) G^{\prime}(0)>O$ and unstable when otherwise.
\end{abstract}

Keywords: Stability, Characteristic Equation, Malaria, Latency.

\section{Introduction}

Malaria is a vector-borne disease. Instead of transmitted directly from human to human, its parasites are transferred between human through mosquitoes. The malaria parasite life cycle is divided into two parts, one is within host (human) body and the other within vector (mosquito) body. Human infection starts from a blood meal of an infectious female mosquito. The parasites existing in the infectious mosquito's saliva, called sporozoites at this stage, enter the bloodstream of the human through mosquito bites and migrate to the liver. Within minutes after entering the human body, sporozoites infect hepatocytes and multiply asexually and asymptomatically in liver for a period of $5-30$ days [1] and [2].

Mathematical models can help understand the dynamics of transmission and spread of the infectious disease and thereby, provide guides and suggestions for the control of the disease. According to Hoshen et al [3], the first person to use mathematical model to quantitatively investigate the spread of malaria was Ross. His model was later extended and studied by Mc-Donald which led to the Ross-McDonald Model [4]-[6].

There are different modeling methodologies which include individual-based models [7], habitat-based models [8], integrated models [9] and [10] and others [11]-[16]. The methodology used in this case is predominantly differential equation-based. Using known clinical and biological information on malaria, a set of differential equation is derived based on the human population subdivided into four compartments of susceptible human $\left(S_{h}\right)$, infected human $\left(I_{h}\right)$, recovered human $\left(R_{h}\right)$ and the therapy class $\left(V_{h}\right)$ while the vector popupation is divided into two compartments of the susceptible vector $\left(S_{V}\right)$ and infected vector $I_{V}$. The endemic equilibrium state is examined for stability.

\section{Model Equations}

The formulation of the model results in the following model equations.

$$
\begin{aligned}
\frac{d S_{h}}{d t} & =\wedge_{h} p+\left(r R_{h}+(\theta-\phi) v_{h}-\mu_{h}-\beta I_{v}\right) s_{h} \\
\frac{d I_{h}}{d t} & =\beta s_{h} I_{v}-\left(\mu_{h}+\mu_{0}+\delta\right) I_{h} \\
\frac{d R_{h}}{d t} & =\delta I_{h}-\left(\mu_{h}-r S_{h}\right) R_{h} \\
\frac{d V_{h}}{d t} & =\wedge_{h}(1-p)+(\phi-\theta) s_{h} v_{h} \\
\frac{d S_{v}}{d t} & =\wedge_{v}-\left(\mu_{v}+e I_{h}\right) s_{v} \\
\frac{d I_{v}}{d t} & =e S_{v} I_{h}-\mu_{v} I_{v}
\end{aligned}
$$


The model consists of six compartments

$S_{h} \quad=\quad$ Susceptible human

$I_{h} \quad=\quad$ Infected human

$R_{h} \quad=\quad$ Recovered human

$S_{V} \quad=\quad$ Susceptible vector

$I_{V} \quad=\quad$ Infected vector

$V_{h} \quad=\quad$ Therapy class

The parameters are

$\Lambda_{V} \quad=\quad$ Birth rate for the vector population

$\Lambda_{h}=$ Birth rate for the human population

$\beta=$ Transmission rate from $\mathrm{S}_{h}$ to $\mathrm{I}_{h}$

$\delta=$ Transmission rate from $\mathrm{I}_{h}$ to $\mathrm{R}_{h}$

$e \quad=\quad$ Transmission rate from $\mathrm{S}_{V}$ to $\mathrm{I}_{V}$

$\theta=$ Transmission rate from $\mathrm{V}_{h}$ to $\mathrm{S}_{h}$

$\phi \quad=\quad$ Transmission rate from $\mathrm{S}_{h}$ to $\mathrm{V}_{h}$

$\mu_{o}=\quad$ Natural death in human population

$\mu_{h} \quad=\quad$ Death resulting from infection in human

$\mu_{V} \quad=\quad$ Natural death in vector population

$r \quad=\quad$ Rate of flow from $\mathrm{R}_{h}$ to $\mathrm{S}_{h}$

$P \quad=\quad$ Recruitment term.

At equilibrium state

\section{Equilibrium State}

$\frac{d S_{h}}{d t}=\frac{d I_{h}}{d t}=\frac{d R_{h}}{d t}=\frac{d V_{h}}{d t}=\frac{d S_{v}}{d t}=\frac{d I_{v}}{d t}=0$

That is

$\wedge_{h} p+\left(r R_{h}+(\theta-\phi) v_{h}-\mu_{h}-\beta I_{v}\right) s_{h}=0$

$\beta s_{h} I_{v}-\left(\mu_{h}+\mu_{0}+\delta\right) I_{h}=0$

$\left(\delta I_{h}-\mu_{h}-r S_{h}\right) R_{h}=0$

$\wedge_{h}(1-p)+(\phi-\theta) s_{h} v_{h}=0$

$\wedge_{v}-\left(\mu_{v}+e I_{h}\right) s_{v}=0$

$e S_{v} I_{h}-\mu_{v} I_{v}=0$

From (12)

$$
s_{v}=\frac{\wedge_{v}}{\mu_{v}+e I_{h}}
$$

From (9)

$$
I_{v}=\frac{\left(\mu_{h}+\mu_{0}+\delta\right) I_{h}}{\beta s_{h}}
$$

Substituting (14) and (15) into (13) and simplifying gives

$$
\begin{aligned}
& I_{h}\left(\frac{e \wedge_{v}}{\mu_{v}+e I_{h}}-\mu_{v} \frac{\left(\mu_{h}+\mu_{0}+\delta\right) I_{h}}{\beta s_{h}}\right)=0 \\
& \frac{e \wedge_{v}}{\mu_{v}+e I_{h}}=\mu_{v} \frac{\left(\mu_{h}+\mu_{0}+\delta\right) I_{h}}{\beta s_{h}}
\end{aligned}
$$

Suppose $I_{h} \neq 0$

Simplifying equation (17) gives

$$
I_{h}=\frac{\beta e \wedge_{v} s_{h}-\mu^{2} v\left(\mu_{h}+\mu_{0}+\delta\right)}{\mu_{v} e\left(\mu_{h}+\mu_{0}+\delta\right)}
$$

From equation (10) 
Suppose $R_{h} \neq 0$

$\delta I_{h}-\mu_{h}-r S_{h}=0$

Put equation (18) into (19)

$\delta\left(\frac{\beta e \wedge_{v} s_{h}-\mu^{2} v\left(\mu_{h}+\mu_{0}+\delta\right)}{\mu_{v} e\left(\mu_{h}+\mu_{0}+\delta\right)}\right)-\mu_{h}-r S_{h}=0$

Simplifying (20) gives

$$
s_{h}=\frac{\left(\mu_{v}+e \mu_{h}\right) \mu_{v} e\left(\mu_{h}+\mu_{0}+\delta\right)}{e^{2}\left(\delta \beta \wedge_{v}-\mu_{v}\left(\mu_{h}+\mu_{0}+\delta\right)\right)}
$$

Substituting (21) into (18) and simplifying

$$
I_{h}=\frac{\left.\beta e^{2} \wedge_{v} \mu_{v}\left(\mu_{v}+e \mu_{h}\right)\left(\mu_{h}+\mu_{0}+\delta\right)\right)}{\left.\mu_{v} e^{3}\left(\delta \beta \wedge_{v}-\mu_{v}\left(\mu_{h}+\mu_{0}+\delta\right)\right)\left(\mu_{h}+\mu_{0}+\delta\right)\right)}-\frac{\left.\mu^{2} v\left(\mu_{h}+\mu_{0}+\delta\right)\right)}{\left.\mu_{v} e\left(\mu_{h}+\mu_{0}+\delta\right)\right)}
$$

Similarly, substituting (22) into (14)

$$
s_{v}=\frac{\wedge_{v}}{\mu_{v}+e\left(\frac{\left.\beta e^{2} \wedge_{v} \mu_{v}\left(\mu_{v}+e \mu_{h}\right)\left(\mu_{h}+\mu_{0}+\delta\right)\right)}{\left.\mu_{v} e^{3}\left(\delta \beta \wedge_{v}-\mu_{v}\left(\mu_{h}+\mu_{0}+\delta\right)\right)\left(\mu_{h}+\mu_{0}+\delta\right)\right)}-\frac{\left.\mu^{2} v\left(\mu_{h}+\mu_{0}+\delta\right)\right)}{\left.\mu_{v} e\left(\mu_{h}+\mu_{0}+\delta\right)\right)}\right)}
$$

Similarly, substituting (21) and (23) into (15)

$$
I_{v}=\frac{\left(\mu_{h}+\mu_{0}+\delta\right) \frac{\left.\beta e^{2} \wedge_{v} \mu_{v}\left(\mu_{v}+e \mu_{h}\right)\left(\mu_{h}+\mu_{0}+\delta\right)\right)}{\left.\mu_{v} e^{3}\left(\delta \beta \wedge_{v}-\mu_{v}\left(\mu_{h}+\mu_{0}+\delta\right)\right)\left(\mu_{h}+\mu_{0}+\delta\right)\right)}-\frac{\left.\mu^{2} v\left(\mu_{h}+\mu_{0}+\delta\right)\right)}{\left.\mu_{v} e\left(\mu_{h}+\mu_{0}+\delta\right)\right)}}{\beta\left(\frac{\left(\mu_{v}+e \mu_{h}\right) \mu_{v} e\left(\mu_{h}+\mu_{0}+\delta\right)}{e^{2}\left(\delta \beta \wedge_{v}-\mu_{v}\left(\mu_{h}+\mu_{0}+\delta\right)\right)}\right)}
$$

Now from equation (11)

$$
\wedge_{h}(1-p)+(\phi-\theta) s_{h} v_{h}=0
$$

Simplifying (25) and making $v_{h}$ the subject

$$
v_{h}=\frac{\wedge_{h}(1-p)}{(\theta-\phi) s_{h}}
$$

Putting (21) into (26)

$$
v_{h}=\frac{\wedge_{h}(1-p)}{(\theta-\phi) \frac{\left(\mu_{v}+e \mu_{h}\right) \mu_{v} e\left(\mu_{h}+\mu_{0}+\delta\right)}{e^{2}\left(\delta \beta \wedge_{v}-\mu_{v}\left(\mu_{h}+\mu_{0}+\delta\right)\right)}}
$$

Also from equation (8)

$$
\wedge_{h} p+\left(r R_{h}+(\theta-\phi) v_{h}-\mu_{h}-\beta I_{v}\right) s=0
$$

Simplifying (28) and making $R_{h}$ the subject

$$
R_{h}=\frac{\mu_{h}+\beta I_{v}}{r}-\frac{(\theta-\phi) v_{h}}{r}-\frac{\wedge_{h} p}{r s_{h}}
$$

Putting (21), (24) and (27) into (29) 


$$
\begin{gathered}
\mu_{h}+\beta\left(\frac{\left(\mu_{h}+\mu_{0}+\delta\right) \frac{\left.\beta e^{2} \wedge_{v} \mu_{v}\left(\mu_{v}+e \mu_{h}\right)\left(\mu_{h}+\mu_{0}+\delta\right)\right)}{\left.\mu_{v} e^{3}\left(\delta \beta \wedge_{v}-\mu_{v}\left(\mu_{h}+\mu_{0}+\delta\right)\right)\left(\mu_{h}+\mu_{0}+\delta\right)\right)}-\frac{\left.\mu^{2} v\left(\mu_{h}+\mu_{0}+\delta\right)\right)}{\left.\mu_{v} e\left(\mu_{h}+\mu_{0}+\delta\right)\right)}}{\beta\left(\frac{\left(\mu_{v}+e \mu_{h}\right) \mu_{v} e\left(\mu_{h}+\mu_{0}+\delta\right)}{e^{2}\left(\delta \beta \wedge_{v}-\mu_{v}\left(\mu_{h}+\mu_{0}+\delta\right)\right)}\right)}\right. \\
\left.R_{h}=\frac{r}{(\theta-\phi) \frac{\wedge_{h}(1-p)}{(\theta-\phi) \frac{\left(\mu_{v}+e \mu_{h}\right) \mu_{v} e\left(\mu_{h}+\mu_{0}+\delta\right)}{e^{2}\left(\delta \beta \wedge_{v}-\mu_{v}\left(\mu_{h}+\mu_{0}+\delta\right)\right)}}}-\frac{\wedge_{h} p}{r}-\frac{\left(\mu_{v}+e \mu_{h}\right) \mu_{v} e\left(\mu_{h}+\mu_{0}+\delta\right)}{e^{2}\left(\delta \beta \wedge_{v}-\mu_{v}\left(\mu_{h}+\mu_{0}+\delta\right)\right)}\right)
\end{gathered}
$$

\section{Stability Analysis of the Endemic Equilibrium}

The characteristic equation is obtained by

$\mathrm{A}=\left|\begin{array}{lccccc}\alpha_{1}-\lambda & 0 & r s_{h} & (\theta-\phi) s_{h} & 0 & -\beta s_{h} \\ \beta I_{v} & -\alpha_{2}-\lambda & 0 & 0 & 0 & \beta s_{h} \\ -r R_{h} & \delta R_{h} & \alpha_{3}-\lambda & 0 & 0 & 0 \\ (\theta-\phi) v_{h} & 0 & 0 & \alpha_{4}-\lambda & 0 & 0 \\ 0 & -e s_{v} & 0 & 0 & \alpha_{5}-\lambda & 0 \\ 0 & e s_{v} & 0 & 0 & e I_{h} & -\mu_{v}-\lambda\end{array}\right|=0$

Which gives

$$
\begin{aligned}
& -\left(-r \lambda^{3}+\left(r \alpha_{5}+\delta \beta I_{v}-r\left(\mu+\alpha_{2}\right)\right) \lambda^{2}+\left(r e s_{v} \beta s_{h}+\left(-\beta \delta I_{v}+r\left(\mu+\alpha_{2}\right)\right) \alpha_{5}+\right.\right. \\
& \left.\left.\left(-r \alpha_{2}+\beta \delta I_{v}\right) \mu\right) \lambda-r e \beta s_{v}\left(e I_{h}+\alpha_{5}\right) s_{h}-\alpha_{5}\left(-r \alpha_{2}+\delta \beta I_{v}\right) \mu\right)\left(-\alpha_{4}+\lambda\right) R_{h}\left(r s_{h}\right) \\
& +\left(-\alpha_{3}+\lambda\right)\left(\lambda^{5}+\left(\mu-\alpha_{4}-\alpha_{1}+\alpha_{2}-\alpha_{5}\right) \lambda^{4}+\left(\left(-\beta e s_{v}-v_{h}(-\theta+\phi)^{2}\right) s_{h}\right.\right. \\
& \left.+\left(-\mu+\alpha_{1}+\alpha_{4}-\alpha_{2}\right) \alpha_{5}+\left(\alpha_{2}-\alpha_{4}-\alpha_{1}\right) \mu+\left(\alpha_{1}-\alpha_{2}\right) \alpha_{4}-\alpha_{1} \alpha_{2}\right) \lambda^{3} \\
& +\left(\left(\left(\beta e s_{v}+v_{h}(-\theta+\phi)^{2} \alpha_{5}+\beta^{2} I_{v} e s_{v}+e\left(e I_{h}+\alpha_{1}+\alpha_{4}\right) \beta s_{v}-v_{h}(-\theta+\phi)^{2}\left(\mu+\alpha_{2}\right)\right) s_{h}\right.\right. \\
& \left.+\left(\left(-\alpha_{2}+\alpha_{4}+\alpha_{1}\right) \mu+\left(-\alpha_{1}+\alpha_{2}\right) \alpha_{4}+\alpha_{1} \alpha_{2}\right) \alpha_{5}+\left(\left(\alpha_{1}-\alpha_{2}\right) \alpha_{4}-\alpha_{1} \alpha_{2}\right) \mu+\alpha_{1} \alpha_{1} \alpha_{4}\right) \lambda^{2} \\
& +\left(\beta e s_{v} v_{h}(\theta+\phi)^{2} s^{2} h+\left(\left(-\beta^{2} I_{v} e s_{v}-e s_{v}\left(\alpha_{1}+\alpha_{4}\right) \beta+v_{h}(-\theta+\phi)^{2}\left(\mu+\alpha_{2}\right)\right) \alpha_{5}\right.\right. \\
& \left.-e s_{v}\left(-e v_{h}+I_{v} \alpha_{4}\right) \beta^{2}-e\left(\left(e I_{h}+\alpha_{1}\right) \alpha_{4}+e I_{h} \alpha_{1}\right) \beta s_{v}-\mu v_{h} \alpha_{2}(-\theta+\phi)^{2}\right) s_{h} \\
& \left.+\left(\left(\left(-\alpha_{1}+\alpha_{2}\right) \alpha_{4}+\alpha_{1} \alpha_{2}\right) \mu-\alpha_{1} \alpha_{2} \alpha_{4}\right) \alpha_{5}+\alpha_{1} \alpha_{2} \alpha_{4} \mu\right) \lambda-(\theta+\phi)^{2} \beta e s_{v}\left(e I_{h}+\alpha_{5}\right) v_{h} s_{h}{ }^{2} \\
& \left.+\left(\left(\beta^{2} I_{v} e s_{v} \alpha_{4}+\alpha_{1} e s_{v} \beta \alpha_{4}+v_{h} \mu \alpha_{2}(-\theta+\phi)^{2}\right) \alpha_{5}+e^{2} \alpha_{4} \beta s_{v}\left(-\beta v_{h}+\alpha_{1}\right)\right) s_{h}-\alpha_{1} \alpha_{2} \alpha_{4} \alpha_{5} \mu\right) \\
& \left.\quad \alpha_{1}=r R_{h}+(\theta-\phi) v_{h}-\mu_{h}-\beta I_{v}\right) \\
& \quad \alpha_{2}=-\left(\mu_{h}+\mu_{0}+\delta\right) \\
& \quad \alpha_{3}=\delta I_{h}-\mu_{h}-r S_{h} \\
& \quad \alpha_{4}=\left((\theta-\phi) s_{h}\right) \\
& \quad \alpha_{5}=\left(\mu_{v}+e I_{h}\right)
\end{aligned}
$$

Bellman and Cooke's theorem is used to establish the stability or otherwise of the model.

\section{Theorem}

Let $H(Z)=P\left(z, e^{z}\right)$ where $p(z, w)$ is a polynomial with principal term.

Suppose $H(i y), y \in R$, is separated into its real and imaginary parts,

$$
H(z)=F(y)+i G(y)
$$


If all zeros of $H(z)$ have negative real parts, then the zeros of $F(y)$ and $G(y)$ are real, simple and alternate and

$G^{\prime}(0) F(0)-G(0) F^{\prime}(0)>0$ for all $y \in R$.

Conversely, all zeros of $H(y)$ will be in the left-half plane provided that either of the following conditions is satisfied:

(i) All the zeros of $F(y)$ and $G(y)$ are real, simple, and alternate and the inequality (34) is satisfied for at least one $\mathrm{y}$.

(ii) All the zeros of $F(y)$ are real and, for each zero, the relation (34) is satisfied

(iii) All the zeros of $G(y)$ are real and, for each zero, the relation (33) is satisfied.

From (32) let

$\mathrm{H}(\lambda)$

$$
\begin{aligned}
& -\left(-r \lambda^{3}+\left(r \alpha_{5}+\delta \beta I_{v}-r\left(\mu+\alpha_{2}\right)\right) \lambda^{2}+\left(\operatorname{res}_{v} \beta s_{h}+\left(-\beta \delta I_{v}+r\left(\mu+\alpha_{2}\right)\right) \alpha_{5}\right.\right. \\
& \left.\left.+\left(-r \alpha_{2}+\beta \delta_{v}\right) \mu\right) \lambda-r e \beta s_{v}\left(e I_{h}+\alpha_{5}\right) s_{h}-\alpha_{5}\left(-r \alpha_{2}+\delta \beta I_{v}\right) \mu\right)\left(-\alpha_{4}+\lambda\right) R_{h}\left(r s_{h}\right) \\
& +\left(-\alpha_{3}+\lambda\right)\left(\lambda^{5}+\left(\mu-\alpha_{4}-\alpha_{1}+\alpha_{2}-\alpha_{5}\right) \lambda^{4}+\left(\left(-\beta e s_{v}-v_{h}(-\theta+\phi)^{2}\right) s_{h}+\right.\right. \\
& \left.\left(-\mu+\alpha_{1}+\alpha_{4}-\alpha_{2}\right) \alpha_{5}+\left(\alpha_{2}-\alpha_{4}-\alpha_{1}\right) \mu+\left(\alpha_{1}-\alpha_{2}\right) \alpha_{4}-\alpha_{1} \alpha_{2}\right) \lambda^{3} \\
= & +\left(\left(\beta e s_{v}+v_{h}(-\theta+\phi)^{2} \alpha_{5}+\beta^{2} I_{v} e s_{v}+e\left(e I_{h}+\alpha_{1}+\alpha_{4}\right) \beta s_{v}-v_{h}(-\theta+\phi)^{2}\left(\mu+\alpha_{2}\right)\right) s_{h}\right. \\
& \left.+\left(\left(-\alpha_{2}+\alpha_{4}+\alpha_{1}\right) \mu+\left(-\alpha_{1}+\alpha_{2}\right) \alpha_{4}+\alpha_{1} \alpha_{2}\right) \alpha_{5}+\left(\left(\alpha_{1}-\alpha_{2}\right) \alpha_{4}-\alpha_{1} \alpha_{2}\right) \mu+\alpha_{1} \alpha_{1} \alpha_{4}\right) \lambda^{2} \\
& +\left(\beta e s_{v} v_{h}(\theta+\phi)^{2} s^{2} h+\left(\left(-\beta^{2} I_{v} e s_{v}-e s_{v}\left(\alpha_{1}+\alpha_{4}\right) \beta+v_{h}(-\theta+\phi)^{2}\left(\mu+\alpha_{2}\right)\right) \alpha_{5}\right.\right. \\
& \left.-e s_{v}\left(-e v_{h}+I_{v} \alpha_{4}\right) \beta^{2}-e\left(\left(e I_{h}+\alpha_{1}\right) \alpha_{4}+e I_{h} \alpha_{1}\right) \beta s_{v}-\mu v_{h} \alpha_{2}(-\theta+\phi)^{2}\right) s_{h}+ \\
& \left.\left(\left(\left(-\alpha_{1}+\alpha_{2}\right) \alpha_{4}+\alpha_{1} \alpha_{2}\right) \mu-\alpha_{1} \alpha_{2} \alpha_{4}\right) \alpha_{5}+\alpha_{1} \alpha_{2} \alpha_{4} \mu\right) \lambda-(\theta+\phi)^{2} \beta e s_{v}\left(e I_{h}+\alpha_{5}\right) v_{h} s_{h}{ }^{2}+ \\
& \left.\left(\left(\beta^{2} I_{v} e s_{v} \alpha_{4}+\alpha_{1} e s_{v} \beta \alpha_{4}+v_{h} \mu \alpha_{2}(-\theta+\phi)^{2}\right) \alpha_{5}+e^{2} \alpha_{4} \beta s_{v}\left(-\beta v_{h}+\alpha_{1}\right)\right) s_{h}-\alpha_{1} \alpha_{2} \alpha_{4} \alpha_{5} \mu\right)
\end{aligned}
$$

Simplifying in powers of $(\lambda)$

$\mathrm{H}(\lambda)=$

$$
\begin{aligned}
& \lambda^{6}+\left(-\alpha_{3}+\mu-\alpha_{4}-\alpha_{1}+\alpha_{2}-\alpha_{5}\right) \lambda^{5}+\left(r R_{h}\left(r s_{h}\right)\right. \\
& +\left(-e s_{v} \beta-v_{h}(-\theta+\phi)^{2}\right) s_{h_{2}}+\left(-\mu+\alpha_{1}+\alpha_{4}-\alpha_{2}\right) \alpha_{5} \\
& \left.+\left(\alpha_{2}-\alpha_{4}-\alpha_{1}\right) \mu+\left(\alpha_{1}-\alpha_{2}\right) \alpha_{4}-\alpha_{1} \alpha_{2}-\alpha_{3}\left(\mu-\alpha_{4}-\alpha_{1}+\alpha_{2}-\alpha_{5}\right)\right) \lambda^{4}+ \\
& \left(\left(\left(\beta e s_{v}+v_{h}(-\theta+\phi)^{2}\right) \alpha_{5}+\beta^{2} I_{v} e s_{v}+e\left(e I_{h}+\alpha_{1}+\alpha_{4}\right) \beta s_{v}\right.\right. \\
& \left.\left.-v_{h}(-\theta+\phi)^{2}\right)\left(\mu+\alpha_{2}\right)\right) s_{h}+\left(\left(-\alpha_{2}+\alpha_{4}+\alpha_{1}\right) \mu+\left(-\alpha h_{1}+\alpha_{2}\right) \alpha_{4}+\alpha_{1} \alpha_{2}\right) \alpha_{5}+ \\
& \left(\left(\alpha_{1}-\alpha_{2}\right) \alpha_{4}-\alpha_{1} \alpha_{2}\right) \mu+\alpha_{1} \alpha_{2}-\alpha_{3}\left(\left(-\beta e s_{v}-v_{n}(-\theta+\phi)^{2}\right) s_{n}\right. \\
& \left.+\left(-\mu+\alpha_{1}+\alpha_{4}-\alpha_{2}\right) \alpha_{5}+\left(\alpha_{2}-\alpha_{4}-\alpha_{1}\right) \mu+\left(\alpha_{1}-\alpha_{2}\right) \alpha_{4}-\alpha_{1} \alpha_{2}\right) \\
& \left.-\left(r \alpha_{5}+\delta A_{v}-r\left(\mu+\alpha_{2}\right)+r \alpha_{4}\right) R_{h}\left(r s_{h}\right)\right) \lambda^{3}+\left(e s_{v} \beta v_{h}(-\theta+\phi)^{2}\right) s_{h}^{2} \\
& +\left(\left(-\beta^{2} I_{v} e s_{v}-e s_{v}\left(\alpha_{1}+\alpha_{4}\right) \beta+v_{h}(-\theta+\phi)^{2}\left(\mu+\alpha_{2}\right)\right) \alpha_{5}-e s_{v}\left(-I_{h} \alpha_{4}\right) \beta^{2}\right. \\
& \left.-e\left(\left(e I_{h}+\alpha_{1}\right) \alpha_{4}+e I_{h} \alpha_{1}\right) \beta s_{v}-v_{h} \mu \alpha_{1}(-\theta+\phi)^{2}\right) s_{h}\left(\left(\left(-\alpha_{1}+\alpha_{2}\right) \alpha_{4}\right.\right. \\
& \left.\left.+\alpha_{1} \alpha_{2}\right) \mu-\alpha_{1} \alpha_{2} \alpha_{4}\right) \alpha_{5}+\alpha_{1} \alpha_{2} \alpha_{4} \mu-\alpha_{3}\left(\left(\left(e s_{v} \beta+v_{h}(-\theta+\phi)^{2}\right) \alpha_{5}\right.\right. \\
& \left.\left.+\beta^{2} I_{v} e s_{v}+e\left(e I_{h}+\alpha_{1}+\alpha_{4}\right) \beta s_{v}-v_{h_{h}}(-\theta+\phi)^{2}\right)\left(\mu+\alpha_{2}\right)\right) s_{h} \\
& +\left(\left(-\alpha_{2}+\alpha_{4}+\alpha_{1}\right) \mu+\left(-\alpha_{1}+\alpha_{2}\right) \alpha_{4}+\alpha_{1} \alpha_{2}\right) \alpha_{5}+\left(\left(\alpha_{1}-\alpha_{2}\right) \alpha_{4}\right. \\
& \left.\left.-\alpha_{1} \alpha_{2}\right) \mu+\alpha_{1} \alpha_{2} \alpha_{4}\right)-\left(\text { res }_{v} \beta s_{h_{h}}+\left(-\delta \beta_{v}+r\left(\mu+\alpha_{2}\right)\right) \alpha_{5}+\right. \\
& \left.\left.\left(-r \alpha_{2}+\delta \boldsymbol{\beta}_{v}-r\left(\mu+\alpha_{2}\right)\right) \alpha_{4}\right) R_{h_{h}}\left(r s_{h_{h}}\right)\right) \lambda^{2}+\left(-\alpha_{3}\left(e s_{v} \beta v_{h}(-\theta+\phi)^{2} s_{h_{h}}^{2}\right.\right. \\
& +\left(\left(-\beta^{2} I_{v} e s_{v}-e s_{v}\left(\alpha_{1}+\alpha_{4}\right) \beta+v_{h}(-\theta+\phi)^{2}\left(\mu+\alpha_{2}\right)\right) \alpha_{5}\right. \\
& \left.-e s_{v}\left(-e I_{h} \alpha_{4}\right) \beta^{2}-e\left(\left(e I_{h}+\alpha_{1}\right) \alpha_{4}+e I_{h} \alpha_{1}\right) \beta s_{v}-v_{h} \mu \alpha_{2}(-\theta+\phi)^{2}\right) s_{h} \\
& \left.\left.\left(\left(\left(-\alpha_{1}+\alpha_{2}\right) \alpha_{4}+\alpha_{1} \alpha_{2}\right) \mu-\alpha_{1} \alpha_{2} \alpha_{4}\right) \alpha_{5}+\alpha_{1} \alpha_{2} \alpha_{4} \mu\right)-(-\theta+\phi)^{2}\right) \alpha_{5}+ \\
& \left.\left.\left.e^{2} \alpha_{4} \beta s_{v}\left(-\beta I_{v} \alpha_{1}\right)\right) s_{h}-\alpha_{1} \alpha_{2} \alpha_{4} \alpha_{5} \mu-\left(-\alpha_{5}\left(-r \alpha_{2}+\delta \beta \Omega_{v}\right) \mu\right) \alpha_{4}\right) R_{h}\left(r s_{h}\right)\right) \lambda \\
& +\left(-\alpha_{5}\left(-r \alpha_{2}+\delta \beta_{v}\right) \mu-r e \beta s_{v}\left(e I_{h}+\alpha_{5}\right) s_{h_{h}}\right) \alpha_{4} R_{h}\left(r s_{h}\right) \\
& -\alpha_{3}(-\theta+\phi)^{2} e \beta s_{v} e I_{h} \alpha_{5} v_{h} s_{h}^{2}+\left(\left(\beta^{2} I_{v} e s_{v} \alpha_{4}+\alpha_{1} e s_{v} \beta \alpha_{4}\right.\right. \\
& +v_{h} \mu \alpha_{2}(-\theta+\phi)^{2} \alpha_{5}+e^{2} \alpha_{4} \beta s_{v}\left(-\beta I_{v} \alpha_{1}\right) s_{h_{2}}-\alpha_{1} \alpha_{2} \alpha_{4} \alpha_{5} \mu
\end{aligned}
$$


Let $\lambda=i p$

$\mathrm{H}(\mathrm{ip})=$

$$
\begin{aligned}
& (i p)^{6}+\left(-\alpha_{3}+\mu-\alpha_{4}-\alpha_{1}+\alpha_{2}-\alpha_{5}\right)(i p)^{5}+\left(r R_{h}\left(r s_{h}\right)\right. \\
& +\left(-e s_{v} \beta-v_{h}(-\theta+\phi)^{2}\right) s_{h}+\left(-\mu+\alpha_{1}+\alpha_{4}-\alpha_{2}\right) \alpha_{5} \\
& \left.+\left(\alpha_{2}-\alpha_{4}-\alpha_{1}\right) \mu+\left(\alpha_{1}-\alpha_{2}\right) \alpha_{4}-\alpha_{1} \alpha_{2}-\alpha_{3}\left(\mu-\alpha_{4}-\alpha_{1}+\alpha_{2}-\alpha_{5}\right)\right)(i p)^{4} \\
& +\left(\left(\left(\beta e s_{v}+v_{h}(-\theta+\phi)^{2}\right) \alpha_{5}+\beta^{2} I_{v} e s_{v}+e\left(e I_{h}+\alpha_{1}+\alpha_{4}\right) \beta s_{v}-v_{h}(-\theta+\phi)^{2}\right)\left(\mu+\alpha_{2}\right)\right) s_{h} \\
& +\left(\left(-\alpha_{2}+\alpha_{4}+\alpha_{1}\right) \mu+\left(-\alpha_{1}+\alpha_{2}\right) \alpha_{4}+\alpha_{1} \alpha_{2}\right) \alpha_{5}+\left(\left(\alpha_{1}-\alpha_{2}\right) \alpha_{4}-\alpha_{1} \alpha_{2}\right) \mu+\alpha_{1} \alpha_{2}- \\
& \alpha_{3}\left(\left(-\beta e s_{v}-v_{h}(-\theta+\phi)^{2}\right) s_{h}+\left(-\mu+\alpha_{1}+\alpha_{4}-\alpha_{2}\right) \alpha_{5}+\left(\alpha_{2}-\alpha_{4}-\alpha_{1}\right) \mu\right. \\
& \left.\left.+\left(\alpha_{1}-\alpha_{2}\right) \alpha_{4}-\alpha_{1} \alpha_{2}\right)-\left(r \alpha_{5}+\delta \beta I_{v}-r\left(\mu+\alpha_{2}\right)+r \alpha_{4}\right) R_{h}\left(r s_{h}\right)\right)(i p)^{3} \\
& +\left(e s_{v} \beta v_{h}(-\theta+\phi)^{2}\right) s_{h}{ }^{2}+\left(\left(-\beta^{2} I_{v} e s_{v}-e s_{v}\left(\alpha_{1}+\alpha_{4}\right) \beta+v_{h}(-\theta+\phi)^{2}\left(\mu+\alpha_{2}\right)\right) \alpha_{5}\right. \\
& \left.-e s_{v}\left(-I_{h} \alpha_{4}\right) \beta^{2}-e\left(\left(e I_{h}+\alpha_{1}\right) \alpha_{4}+e I_{h} \alpha_{1}\right) \beta s_{v}-v_{h} \mu \alpha_{1}(-\theta+\phi)^{2}\right) s_{h}\left(\left(\left(-\alpha_{1}+\alpha_{2}\right) \alpha_{4}+\alpha_{1} \alpha_{2}\right) \mu\right. \\
& \left.-\alpha_{1} \alpha_{2} \alpha_{4}\right) \alpha_{5}+\alpha_{1} \alpha_{2} \alpha_{4} \mu-\alpha_{3}\left(\left(\left(e s_{v} \beta+v_{h}(-\theta+\phi)^{2}\right) \alpha_{5}+\beta^{2} I_{v} e s_{v}+e\left(e I_{h}+\alpha_{1}+\alpha_{4}\right) \beta s_{v}\right.\right. \\
& \left.\left.-v_{h}(-\theta+\phi)^{2}\right)\left(\mu+\alpha_{2}\right)\right) s_{h}+\left(\left(-\alpha_{2}+\alpha_{4}+\alpha_{1}\right) \mu+\left(-\alpha_{1}+\alpha_{2}\right)\right. \\
& \left.\left.\alpha_{4}+\alpha_{1} \alpha_{2}\right) \alpha_{5}+\left(\left(\alpha_{1}-\alpha_{2}\right) \alpha_{4}-\alpha_{1} \alpha_{2}\right) \mu+\alpha_{1} \alpha_{2} \alpha_{4}\right)-\left(\operatorname{res}_{v} \beta s_{h}+\left(-\delta \beta I_{v}+r\left(\mu+\alpha_{2}\right)\right) \alpha_{5}\right. \\
& \left.\left.+\left(-r \alpha_{2}+\delta \beta I_{v}-r\left(\mu+\alpha_{2}\right)\right) \alpha_{4}\right) R_{h}\left(r s_{h}\right)\right)(i p)^{2}+\left(-\alpha_{3}\left(e s_{v} \beta v_{h}(-\theta+\phi)^{2} s_{h}{ }^{2}\right.\right. \\
& +\left(\left(-\beta^{2} I_{v} e s_{v}-e s_{v}\left(\alpha_{1}+\alpha_{4}\right) \beta+v_{h}(-\theta+\phi)^{2}\left(\mu+\alpha_{2}\right)\right) \alpha_{5}-e s_{v}\left(-e I_{h} \alpha_{4}\right) \beta^{2}\right. \\
& \left.-e\left(\left(e I_{h}+\alpha_{1}\right) \alpha_{4}++e I_{h} \alpha_{1}\right) \beta s_{v}-v_{h} \mu \alpha_{2}(-\theta+\phi)^{2}\right) s_{h}\left(\left(\left(-\alpha_{1}+\alpha_{2}\right) \alpha_{4}+\alpha_{1} \alpha_{2}\right) \mu\right. \\
& \left.\left.\left.\left.-\alpha_{1} \alpha_{2} \alpha_{4}\right) \alpha_{5}+\alpha_{1} \alpha_{2} \alpha_{4} \mu\right)-(-\theta+\phi)^{2}\right) \alpha_{5}+e^{2} \alpha_{4} \beta s_{v}\left(-\beta I_{v} \alpha_{1}\right)\right) s_{h}-\alpha_{1} \alpha_{2} \alpha_{4} \alpha_{5} \mu \\
& \left.\left.-\left(-\alpha_{5}\left(-r \alpha_{2}+\delta \beta I_{v}\right) \mu\right) \alpha_{4}\right) R_{h}\left(r s_{h}\right)\right)(i p)+\left(-\alpha_{5}\left(-r \alpha_{2}+\delta \beta I_{v}\right) \mu-r e \beta s_{v}\left(e I_{h}+\alpha_{5}\right) s_{h}\right) \alpha_{4} R_{h}\left(r s_{h}\right) \\
& -\alpha_{3}(-\theta+\phi)^{2} e \beta s_{v} e I_{h} \alpha_{5} v_{h} s_{h}{ }^{2}+\left(\left(\beta^{2} I_{v} e s_{v} \alpha_{4}+\alpha_{1} e s_{v} \beta \alpha_{4}+v_{h} \mu \alpha_{2}(-\theta+\phi)^{2} \alpha_{5}\right.\right. \\
& \left.+e^{2} \alpha_{4} \beta s_{v}\left(-\beta I_{v} \alpha_{1}\right)\right) s_{h}-\alpha_{1} \alpha_{2} \alpha_{4} \alpha_{5} \mu
\end{aligned}
$$

Resolving into real and imaginary

$$
\begin{aligned}
& H(i p)=F(p)+i G(p) \\
& F(p)=(-p)^{6}+\left(r R_{h}\left(r s_{h}\right)+\left(-e s_{v} \beta-v_{h}(-\theta+\phi)^{2}\right) s_{h}+\right. \\
& \left(-\mu+\alpha_{1}+\alpha_{4}-\alpha_{2}\right) \alpha_{5}+\left(\alpha_{2}-\alpha_{4}-\alpha_{1}\right) \mu+\left(\alpha_{1}-\alpha_{2}\right) \alpha_{4} \\
& \left.-\alpha_{1} \alpha_{2}-\alpha_{3}\left(\mu-\alpha_{4}-\alpha_{1}+\alpha_{2}-\alpha_{5}\right)\right)(p)^{4}+\left(e s_{v} \beta v_{h}(-\theta+\phi)^{2}\right) s_{h}{ }^{2} \\
& +\left(\left(-\beta^{2} I_{v} e s_{v}-e s_{v}\left(\alpha_{1}+\alpha_{4}\right) \beta+v_{h}(-\theta+\phi)^{2}\left(\mu+\alpha_{2}\right)\right) \alpha_{5}-e s_{v}\left(-I_{h} \alpha_{4}\right) \beta^{2}\right. \\
& \left.-e\left(\left(e I_{h}+\alpha_{1}\right) \alpha_{4}+e I_{h} \alpha_{1}\right) \beta s_{v}-v_{h} \mu \alpha_{1}(-\theta+\phi)^{2}\right) s_{h}\left(\left(\left(-\alpha_{1}+\alpha_{2}\right) \alpha_{4}+\alpha_{1} \alpha_{2}\right) \mu\right. \\
& \left.-\alpha_{1} \alpha_{2} \alpha_{4}\right) \alpha_{5}+\alpha_{1} \alpha_{2} \alpha_{4} \mu-\alpha_{3}\left(\left(\left(e s_{v} \beta+v_{h}(-\theta+\phi)^{2}\right) \alpha_{5}+\beta^{2} I_{v} e s_{v}\right.\right. \\
& \left.\left.+e\left(e I_{h}+\alpha_{1}+\alpha_{4}\right) \beta s_{v}-v_{h}(-\theta+\phi)^{2}\right)\left(\mu+\alpha_{2}\right)\right) s_{h}+\left(\left(-\alpha_{2}+\alpha_{4}+\alpha_{1}\right) \mu+\left(-\alpha_{1}+\alpha_{2}\right)\right. \\
& \left.\left.\alpha_{4}+\alpha_{1} \alpha_{2}\right) \alpha_{5}+\left(\left(\alpha_{1}-\alpha_{2}\right) \alpha_{4}-\alpha_{1} \alpha_{2}\right) \mu+\alpha_{1} \alpha_{2} \alpha_{4}\right)-\left(r e s_{v} \beta s_{h}+\left(-\delta \beta I_{v}+r\left(\mu+\alpha_{2}\right)\right) \alpha_{5}+\right. \\
& \left.\left.\left(-r \alpha_{2}+\delta \beta I_{v}-r\left(\mu+\alpha_{2}\right)\right) \alpha_{4}\right) R_{h}\left(r s_{h}\right)\right)(-p)^{2}+\left(-\alpha_{5}\left(-r \alpha_{2}+\delta \beta I_{v}\right) \mu-r e \beta s_{v}\left(e I_{h}+\alpha_{5}\right) s_{h}\right) \alpha_{4} R_{h} \\
& \left(r s_{h}\right)-\alpha_{3}(-\theta+\phi)^{2} e \beta s_{v} e I_{h} \alpha_{5} v_{h} s_{h}{ }^{2}+\left(\left(\beta^{2} I_{v} e s_{v} \alpha_{4}+\alpha_{1} e s_{v} \beta \alpha_{4}+v_{h} \mu \alpha_{2}(-\theta+\phi)^{2} \alpha_{5}\right.\right. \\
& \left.+e^{2} \alpha_{4} \beta s_{v}\left(-\beta I_{v} \alpha_{1}\right)\right) s_{h}-\alpha_{1} \alpha_{2} \alpha_{4} \alpha_{5} \mu
\end{aligned}
$$




$$
\begin{aligned}
& G(p)=\left(-\alpha_{3}+\mu-\alpha_{4}-\alpha_{1}+\alpha_{2}-\alpha_{5}\right)(p)^{5}+\left(\left(\left(\beta e s_{v}+v_{h}(-\theta+\phi)^{2}\right) \alpha_{5}+\beta^{2} I_{v} e s_{v}+\right.\right. \\
& \left.\left.e\left(e I_{h}+\alpha_{1}+\alpha_{4}\right) \beta s_{v}-v_{h}(-\theta+\phi)^{2}\right)\left(\mu+\alpha_{2}\right)\right) s_{h}+\left(\left(-\alpha_{2}+\alpha_{4}+\alpha_{1}\right) \mu+\left(-\alpha_{1}+\alpha_{2}\right) \alpha_{4}+\alpha_{1} \alpha_{2}\right) \alpha_{5}+ \\
& \left(\left(\alpha_{1}-\alpha_{2}\right) \alpha_{4}-\alpha_{1} \alpha_{2}\right) \mu+\alpha_{1} \alpha_{2}-\alpha_{3}\left(\left(-\beta e s_{v}-v_{h}(-\theta+\phi)^{2}\right) s_{h}+\left(-\mu+\alpha_{1}+\alpha_{4}-\alpha_{2}\right) \alpha_{5}+\right. \\
& \left.\left.\left(\alpha_{2}-\alpha_{4}-\alpha_{1}\right) \mu+\left(\alpha_{1}-\alpha_{2}\right) \alpha_{4}-\alpha_{1} \alpha_{2}\right)-\left(r \alpha_{5}+\delta \beta I_{v}-r\left(\mu+\alpha_{2}\right)+r \alpha_{4}\right) R_{h}\left(r s_{h}\right)\right)(-p)^{3} \\
& +\left(-\alpha_{3}\left(e s_{v} \beta v_{h}(-\theta+\phi)^{2} s_{h}^{2}+\left(\left(-\beta^{2} I_{v} e s_{v}-e s_{v}\left(\alpha_{1}+\alpha_{4}\right) \beta+v_{h}(-\theta+\phi)^{2}\left(\mu+\alpha_{2}\right)\right) \alpha_{5}\right.\right.\right. \\
& \left.-e s_{v}\left(-e I_{h} \alpha_{4}\right) \beta^{2}-e\left(\left(e I_{h}+\alpha_{1}\right) \alpha_{4}++e I_{h} \alpha_{1}\right) \beta s_{v}-v_{h} \mu \alpha_{2}(-\theta+\phi)^{2}\right) s_{h}\left(\left(\left(-\alpha_{1}+\alpha_{2}\right) \alpha_{4}+\alpha_{1} \alpha_{2}\right) \mu\right. \\
& \left.\left.\left.\left.-\alpha_{1} \alpha_{2} \alpha_{4}\right) \alpha_{5}+\alpha_{1} \alpha_{2} \alpha_{4} \mu\right)-(-\theta+\phi)^{2}\right) \alpha_{5}+e^{2} \alpha_{4} \beta s_{v}\left(-\beta I_{v} \alpha_{1}\right)\right) s_{h}-\alpha_{1} \alpha_{2} \alpha_{4} \alpha_{5} \mu- \\
& \left.\left.\left(-\alpha_{5}\left(-r \alpha_{2}+\delta \beta I_{v}\right) \mu\right) \alpha_{4}\right) R_{h}\left(r s_{h}\right)\right)(p)
\end{aligned}
$$

Differentiating (39) and (40) with respect to $\mathrm{p}$ and setting $p=0$

$$
\begin{aligned}
& F^{\prime}(0)=0 \\
& G^{\prime}(0)=\left(-\alpha_{3}\left(e s_{v} \beta v_{h}(-\theta+\phi)^{2} s_{h}{ }^{2}+\left(\left(-\beta^{2} I_{v} e s_{v}-e s_{v}\left(\alpha_{1}+\alpha_{4}\right) \beta\right.\right.\right.\right. \\
& \left.+v_{h}(-\theta+\phi)^{2}\left(\mu+\alpha_{2}\right)\right) \alpha_{5}-e s_{v}\left(-e I_{h} \alpha_{4}\right) \beta^{2}-e\left(\left(e I_{h}+\alpha_{1}\right) \alpha_{4}\right. \\
& \left.\left.+e I_{h} \alpha_{1}\right) \beta s_{v}-v_{h} \mu \alpha_{2}(-\theta+\phi)^{2}\right) s_{h}\left(\left(\left(-\alpha_{1}+\alpha_{2}\right) \alpha_{4}+\alpha_{1} \alpha_{2}\right) \mu\right. \\
& \left.\left.\left.\left.-\alpha_{1} \alpha_{2} \alpha_{4}\right) \alpha_{5}+\alpha_{1} \alpha_{2} \alpha_{4} \mu\right)-(-\theta+\phi)^{2}\right) \alpha_{5}+e^{2} \alpha_{4} \beta s_{v}\left(-\beta I_{v} \alpha_{1}\right)\right) s_{h} \\
& \left.\left.-\alpha_{1} \alpha_{2} \alpha_{4} \alpha_{5} \mu\left(-\alpha_{5}\left(-r \alpha_{2}+\delta \beta I_{v}\right) \mu\right) \alpha_{4}\right) R_{h}\left(r s_{h}\right)\right)
\end{aligned}
$$

Setting $\mathrm{p}=0$ into (39) and (40) gives

$$
\begin{aligned}
& F(0)=\left(-\alpha_{5}\left(-r \alpha_{2}+\delta \beta I_{v}\right) \mu-r e \beta s_{v}\left(e I_{h}+\alpha_{5}\right) s_{h}\right) \alpha_{4} R_{h}\left(r s_{h}\right) \\
& -\alpha_{3}(-\theta+\phi)^{2} e \beta s_{v} e I_{h} \alpha_{5} v_{h} s_{h}{ }^{2}+\left(\left(\beta^{2} I_{v} e s_{v} \alpha_{4}+\alpha_{1} e s_{v} \beta \alpha_{4}+\right.\right. \\
& \left.v_{h} \mu \alpha_{2}(-\theta+\phi)^{2} \alpha_{5}+e^{2} \alpha_{4} \beta s_{v}\left(-\beta I_{v} \alpha_{1}\right)\right) s_{h}-\alpha_{1} \alpha_{2} \alpha_{4} \alpha_{5} \mu \\
& G(0)=0
\end{aligned}
$$

Hence $F(0) G^{\prime}(0)>0$

Let $S_{*}=F(0) G^{\prime}(0)$

Therefore, the non-zero state will be stable when $S_{*}>0$ and unstable if otherwise.

\section{Conclusion}

This paper proposes a mathematical model on the spread of malaria using a system of ordinary differential equations with six compartments. The equilibrium states were obtained and the endemic state analysed for stability. It is observed from the mathematical analysis that the non-zero equilibrium state will be stable when $F(0) G^{\prime}(0)>0$ and unstable otherwise.

\section{References}

[1]. G. H. Bledsoe, Malaria Primer for Clinicians in the United State, South. Med. J1998, 12; Pp 1197-1204.

[2]. J.D. Charlwood, T. Smith, P. F. Billingsley, W. Takken, E. Lyimo, \& J. Meuwissen, Survival and Infection Probabilities of Anthropophagic Anophelines from an Area of High Prevalence of Plasmodium Falciparum in Humans, Bull. Entomol L.Res,, 1997, 87 : Pp. 445-453.

[3]. M. B. Hoshen, R. Heinrich, W. D. Stein, \& H. Ginsburg, Mathematical Modeling of the Within-host Dyamics of Plasmodium Falciparum. Parasitology. 2001, 121: 22-235.

[4]. P. L. Alonso, G. Brown, M. Arevalo, F. Binka, F. Chitnis, F. Collins, O. Doumbo, B. Greenwood, L. Hall, M. Levine, K. Mendis, R. Newmann, C. Plowe, M. H. Rodriguez, R. Sinden, L. Shusker \& M. A. Tanner, Research Agenda to Underpin Malaria Eradication. PloS Med. 8:e1000406, 2011.

[5]. N.T.J.Bailey, The Biomathematics of Malaria. London: Charles Griffin and Co. Ltd 1982

[6]. W.O. Kermack \& A.G. McKendrick, Contribution to the Mathematical Theory to Epidemics. Proc R Soc Lond. Series A. 1922, 155: $100-121$.

[7]. G.F. Killeen, W. Gu, C.M. Mbogo, J.L. Regens, J.I. Githure \& J.C.Beier, An Individual-Based Model of Plasmodium Falciparum Malaria Transmission on the Coast of Kenya. Trans R. Soc. Trop Med. Hyg. 2003, 97: 43-50.

[8]. C.M. Mbogo, W. Gu, J.I. Githure, J.L. Regens, G.F. Killeen, C.M. Swalm, G. Yan \& J.C. Beiser, Low Recovery Rates Stabilize Malaria Endemicity in Areas of Low Transmission in Coastal Kenya. Acta Trop. 2003, 86: 71-81.

[9]. F.E. McKenzie \& W.H. Bossert, An Integrated Model of Plasmodium Falciparum Dynamics. J Theor Biol. 2005, 232: $411-426$. 
[10]. D.L. Smith, J. Dushoff \& F.E. McKenzie, The Risk of Mosquito Borne Infection in a Heterogeneous Environment. Plos Biol. 2004, 2: $1957-1964$

[11]. G.F. Killeen, F.E. McKenzie, B.D. Foy, C. Schieffeling, P.F. Billingsley .\& J.C. Beier, A Simplified Model for Predicting Malaria Entomological Inoculation Rates Based on Entomologic and Parasitologic Parameters Relevant to Control. Am J. Trop. Med. Hyg. 2003, 62: 535-544

[12]. F.E.McKenzie, Why Model Malaria? Parasitol Today. 2000, 16: 458-464.

[13]. T. Smith, N. Maire, A. Ross, M. Penny, N. Chitnis, A. Schapira, A. Studer. B. Cunton, C. Lengeler, F. Tediosi, D. De Savigny \& M. Tanner, Towards a Comprehensive Simulation Model of Malaria Epidemiology and Control. Parasitology. 2008, 135: 15071516 .

[14]. N. Chitnis, J.M. Cushin \& J.M. Hyman, Bifurcation Analysis of a Mathematical Model for Malaria Transmission. SIAM J APPL Math. 2006, 67: 24-45.

[15]. G.A.Ngwa, On the Population Dynamics of the Malaria Vector. Bull Math Biol. 2006, 68: 2161-2189.

[16]. P.E.Parham, \& N.M. Ferguson, Space and Contact Networks: Capturing the Locality of Disease Transmission. JR SOC Interface. 2006, 3: 483-493. 